\title{
Biomonitoreo de la microcirculación durante cirugía cardiovascular
}

\author{
Microcirculation biomonitoring during cardiac surgery \\ Edgardo Banille1, Tania Aguirre², Ramiro Ríos², Irma Azar', Carlos Resino', Gabriel Paz¹, \\ Gabriela Portillo', Magalí Velázquez', Alejandro Peirone ${ }^{3}$
}

\begin{abstract}
Introduction: Microcirculation, which encompasses the blood vessels smaller than 100 microns, works as an interconnected biological system; its main functions are ensuring tissue perfusion and oxygenation. During extracorporeal circulation (CPB), a "systemic inflammatory response" (SIRS) with microcirculatory endothelial dysfunction may occur[1],[2]. Objective: To indirectly evaluate microcirculation, using "tissue oxygenation" markers central venous oxygen saturation $\left(\mathrm{SvCO}_{2}\right)$ oxygen extraction index $\left(\mathrm{O}_{2 \mathrm{E}}\right)$ and the "perfusion markers", as the Delta marker or the venoarterial $\mathrm{CO}_{2}$ difference (Difv-aCO$)$ and arterial and venous lactate concentration. Aside from the tissue perfusion marker, a new marker has been considered: the "respiratory quotient", which is the ratio of the carbon dioxide released to oxygen consumed $\left(\mathrm{Pv}-\mathrm{aCO} \mathrm{CO}_{2} / \mathrm{Ca}-\mathrm{vO}_{2}\right)$. [3],[4],[5]. Methods: Between March 1st and August 1st, 2018, 34 patients with congenital cardiopathies that underwent $\mathrm{CPB}$ surgery were studied. They were divided in groups A: $15 p$ (RASCH score 1 and 2) and B: 19p (RASCH score 3, 4 and 5). Results: Variables derived from oxygen: $\mathrm{SvCO}_{2}$ and $\mathrm{O}_{2} \mathrm{El}$ did not present statistically significative differences, nor did the tissue perfusion marker Dif.v- $\mathrm{aCO}_{2}$. However, the other perfusion markers, such as serum lactate and respiratory quotient $P v-a \mathrm{CO}_{2} / \mathrm{Ca}-\mathrm{vO}_{2}$, showed significant differences $(\mathrm{p}<$ 0,001 ). Conclusions: Respiratory quotient assessment $P v-\mathrm{aCO}_{2} / \mathrm{Ca}-\mathrm{vO}_{2}$, which has been endorsed by numerous publications related to septic shock, proves a great tool to expose hidden shock and as a reliable marker of evolution and adverse effects. Patel y cols[6] show its use on pediatric patients with congenital cardiopathies after CPB surgery. As we replicated their experience, we found
\end{abstract}

\section{Key words:}

Microcirculation, biomonitoring, respiratory quotient

Servicio Terapia Intensiva Cardiovascular Hospital de Niños de la Santísima Trinidad. Servicio de Anestesia Cardiovascular del Hospital de Niños de la Santísima Trinidad. Servicio de Cardiología Pediátrica del Hospital de Niños de la Santísima Trinidad.

Córdoba. Argentina.

Fecha de recepción: 08 de junio de 2020

Fecha de aceptación: 12 de septiembre de 2020

\section{ORCID}

0000-0003-1148-9254

Correspondencia:

Tania Aguirre

aguirretania184@gmail.com 
that this marker is better for identifying low cardiac output, even in early stages, than lactate values.

\section{RESUMEN}

Introducción: La microcirculación comprende los vasos sanguíneos menores de 100 micras, se comporta como una red de trabajo biológico interconectado, donde las principales funciones son asegurar la perfusión y oxigenación tisular. Durante la circulación extracorpórea (CEC), se puede presentar una "Respuesta inflamatoria Sistémica", (SIRS), que genera una disfunción endotelial con alteración microcirculatoria[1],[2]. Objetivo: El objetivo es evaluar indirectamente la microcirculación, utilizando los marcadores de "oxigenación tisular", con las variables derivadas del oxígeno: saturación venosa central de oxígeno $\left(\mathrm{SvCO}_{2}\right)$ y el índice de extracción de oxígeno $\left(\mathrm{IEO}_{2}\right)$ y los "marcadores de perfusión", como el delta o diferencia veno-arterial de $\mathrm{CO}_{2}\left(\right.$ Difv-aCO $\left.\mathrm{CO}_{2}\right)$ y la determinación de lactatos arterial y venoso. Además analizar el nuevo marcador de perfusión tisular: "Cociente respiratorio" o gradiente de presión venoarterial de $\mathrm{CO}_{2} /$ contenido arterio-venoso de $\mathrm{O}_{2:} \mathrm{Pv} \mathrm{aCO} \mathrm{CO}_{2} / \mathrm{Ca}-\mathrm{vO} \mathrm{O}_{2}$.[3],[4],[5]. Material y Métodos: Entre el $1^{\circ}$ de marzo y el 1 de agosto del año 2018), se estudiaron 34 pacientes con cardiopatías congénitas operados bajo circulación extracorpórea. Se dividieron en grupo A: $15 \mathrm{p}$ (score RASCH 1 y 2) y grupo B 19 p (score RASCH 3, 4 y 5). Resultados: Las variables derivadas del oxígeno: $\mathrm{SvCO}_{2}$ y IEO 2 no presentaron diferencias estadísticamente significativas al igual que el marcador de perfusión tisular Dif.v-aCO $\mathrm{CO}_{2}$. En cambio, los otros marcadores de perfusión, como la determinación de lactatos séricos y el cociente respiratorio $\mathrm{Pv}-\mathrm{aCO} \mathrm{CO}_{2} / \mathrm{Ca}-\mathrm{vO}_{2}$, presentaron diferencias significativas $(\mathrm{p}<0,001)$. Conclusiones: La evaluación del nuevo marcador de perfusión "Cociente respiratorio": $\mathrm{PV}-\mathrm{aCO} \mathrm{CO}_{2} / \mathrm{Ca}-\mathrm{VO}_{2}$, es avalada en numerosas publicaciones de shock séptico, donde demuestra una gran utilidad para desenmascarar estados de shock crípticos (oculto) y como marcador de evolución y de efectos adversos. Patel y cols[6] demuestra la utilidad del mismo en pacientes pediátricos con cardiopatías congénitas operados bajo circulación extracorpórea, replicando dicha experiencia, encontramos que dicho marcador muestra un valor promisorio en identificar estados de bajo gasto cardíaco, aún en forma más precoz que la determinación de la curva de lactatos.
Palabras clave:

Microcirculación, biomonitoreo, cociente respiratorio

\section{Introducción}

L

os factores relacionados con la respuesta inflamatoria desencadenada con la circulación extracor-pórea (SIRS post CEC), se vinculan al contacto de la sangre a superficies extrañas del circuito, la hemodilución, los cambios de flujo no pulsátiles durante la CEC a flujos pulsátiles a la salida de bomba, clampeo aórtico, el fenómeno de isquemia/reperfusión, la hipotermia/recalentamiento, los cambios de presiones intrapulmonares durante la asistencia respiratoria mecánica (volutrauma), los tipos de oxigenador, todos estos factores generan la activación de los sistemas de cascada orgánicos (contacto, complemento, coagulación, fibrinólisis) con alteración de las función microcirculatoria[1],[2],[3].

Se analizan los marcadores de oxigenación y perfusión capilar. Considerando en conjunto estas variables, se pueden configurar perfiles hemodinámicos que nos orientan a la toma de decisiones[3],[4],[5],[7],[8] Tabla 1.

\section{Objetivos}

Evaluar indirectamente la microcirculación, utilizan- 


\begin{tabular}{|c|c|c|c|}
\hline Oxigenación tisular & Saturación venosa central $\left(\mathrm{SvcO}_{2}\right)$ & $\begin{array}{l}\text { Índice de extracción de oxí- } \\
\text { geno }\left(\mathrm{IEO}_{2}\right)\end{array}$ & \\
\hline Marcadores de perfusión & Diferencia veno-arterial de $\mathrm{CO}_{2}$ & Lactato arterial & Lactato venoso \\
\hline $\begin{array}{l}\text { Nuevo marcador de perfu- } \\
\text { sión }\end{array}$ & $\begin{array}{l}\text { Cociente respiratorio o gradiente de } \\
\text { presión veno-arterial de } \mathrm{CO}_{2} / \mathrm{conte}- \\
\text { nido a-v de } \mathrm{O}_{2}: \text { (Dif.Pv-aCO } \mathrm{CO}_{2} / \mathrm{Ca}-\mathrm{vO}_{2} \text { ) }\end{array}$ & & \\
\hline
\end{tabular}

do marcadores de "oxigenación tisular", con las variables derivadas del oxígeno: saturación venosa central de oxígeno $\left(\mathrm{SvCO}_{2}\right)$, índice de extracción de oxígeno $\left(\mathrm{IEO}_{2}\right)$ y con "marcadores de perfusión" como el delta o diferencia veno-arterial de $\mathrm{CO}_{2}\left(\right.$ Difv- $\left.\mathrm{aCO}_{2}\right)$ y la determinación de lactatos arterial y venoso. Evaluar el rol de un nuevo marcador de perfusión: Cociente respiratorio o gradiente de presión veno-arterial de $\mathrm{CO}_{2} /$ contenido arterio-venoso de $\mathrm{O}_{2} \mathrm{Pv}-\mathrm{aCO} / \mathrm{Ca}-\mathrm{vO}_{2}$. VN: $<1$.

\section{Material y Métodos}

Estudio prospectivo observacional aprobado por el comité de Docencia y de Ética del Hospital de Niños de la Santísima Trinidad de la Ciudad de Córdoba y consentimiento aprobado por los padres.

Entre el $1^{\circ}$ de marzo y el 1 de agosto del año 2018, se estudiaron 34 pacientes operados bajo circulación extracorpórea. Se registraron variables generales previas a la intervención, como edad, peso, tipo de cardiopatía, técnica quirúrgica y variables intraoperatorias, como tiempo de bomba, tiempo de clampeo aórtico, grado de hipotermia y si se requirió parada circulatoria. Se dividieron en grupo A: 15 p (score RASCH 1 y 2) y grupo B 19 p (score RASCH 3, 4 y 5).

Se realizaron en quirófano vía arterial (arteria radial) y vía venosa central (unión cavo-atrial). Se obtuvieron muestras de gases arteriales y venosos, se realizaron fórmulas de las "variables derivadas del oxígeno y se midieron valores de lactatos y de hemoglobina.

Las muestran se tomaron a los 30 minutos antes del ingreso del paciente a CEC, a los 30 minutos de iniciada la misma y al finalizar la CEC.

\section{Fórmulas}

Contenido arterial de oxígeno: $\mathrm{CaO}_{2}(\mathrm{Hb} \times 1,34 \mathrm{x}$ $\left.\mathrm{SaO}_{2}\right)+\left(\mathrm{PaO}_{2} \times 0,003\right)$

VN: $18-21 \mathrm{~mL} / \mathrm{dL}$

Ejemplo: $\mathrm{CaO}_{2}:(15 \times 1,34 \times 0,98)+(60 \times 0,003)=$
$19.6+0,18=19,78 \mathrm{~mL} / \mathrm{dL}$

Contenido venoso de oxígeno: $\mathrm{CvO}_{2}(\mathrm{Hb} \times 1,34 \mathrm{x}$ $\left.\mathrm{SvO}_{2}\right)+\left(\mathrm{PvO}_{2} \times 0,003\right)$

VN: $12-15 \mathrm{~mL} / \mathrm{dL}$

Ejemplo: $\mathrm{CvO}_{2}=(15 \times 1,34 \times 0,70)+(40 \times 0,003)$ $=14,07+0,12=14,19 \mathrm{~mL} / \mathrm{dL}$

Nota: valor calculado: $15 \mathrm{~g}$ de hemoglobina $(\mathrm{Hb})$

Diferencia de contenido arterio-venoso de oxígeno: $\mathrm{Ca}-\mathrm{vO}_{2}=\mathrm{CaO}_{2}-\mathrm{CvO}_{2}$

VN: 3 a $\mathrm{mL} / \mathrm{dL}$ de sangre. 10

Consumo de oxígeno: $\left(\mathrm{VO}_{2}\right)=\left(\right.$ Dif a- $\left.\mathrm{vO}_{2}\right)(\mathrm{GC}) \mathrm{x}$

Nota: se multiplica por 10 para igualar las unidades de la (Dif a-vO $)$

Entrega o disponibilidad de oxígeno $\left(\mathrm{DO}_{2}\right)=$ $\left(\mathrm{CaO}_{2}\right) \times(\mathrm{GC}) \times 10$

Índice de extracción de oxígeno: $\mathrm{DO}_{2} \mathrm{NO}_{2}$

$\mathrm{IEO}_{2}=\mathrm{SaO}_{2}-\mathrm{SvCO}_{2} / \mathrm{SaO}_{2} \cdot \mathrm{VN}: 25 \% \%$. Ejemplo IEO2 $=100-75 / 100=25 \%$

También se puede calcular: (Dif a- $\left.\mathrm{vO}_{2}\right) \times 100$ 5,59 $=0,28(28 \%)\left(\mathrm{CaO}_{2}\right)(19,78)$

Nota: Si disminuye el $\mathrm{DO}_{2}$, aumenta el índice de $\mathrm{EO}_{2}$ para mantener constante el $\mathrm{VO}_{2}$.

Gasto cardíaco $=$ Dif a-vO $\times 100 / \mathrm{CaO}_{2}=5,59 \times$ $100 / 19,78=559 / 19,78=28=5 \mathrm{~L} / \mathrm{min}$

Dif a-vO , 5,59 5,59

Diferencia o delta de presión parcial veno-arterial de $\mathrm{CO}_{2}:$ Pv-aCO$=\mathrm{PvCO}_{2}-\mathrm{PaCO}_{2}$ :

VN: $5 \mathrm{mmHg}$.

Cociente respiratorio o gradiente de presión veno-arterial de $\mathrm{CO}_{2} /$ contenido arterio-venoso de $\mathrm{O}_{2}$ : $\mathrm{PV}-\mathrm{aCO}_{2} / \mathrm{Ca}-\mathrm{vO}_{2} . \mathrm{VN}:<1$ 


\section{Tabla 2. Distribución de pacientes según Score RASCH y cirugías}

\begin{tabular}{|c|c|c|c|}
\hline Grupo A (RASCH 1 y 2) & & Grupo B (RASCH 3, 4 y 5) & \\
\hline CIA ostium secundum & 7 & Tetralogía de Fallot (TOF) & 3 \\
\hline $\begin{array}{l}\text { CIV perimembranosa } \\
\mathrm{CIC}+\mathrm{CIA}+\mathrm{DAP}\end{array}$ & $\begin{array}{l}5 \\
2\end{array}$ & Coart. de AO + CIV & 2 \\
\hline \multirow[t]{14}{*}{ CANAL AV Transicional } & 1 & AtP tubo de VD-AP & 1 \\
\hline & & AtP cambio tubo VD & 1 \\
\hline & & CIV + Estenosis subAo & 1 \\
\hline & & Cirugía de Kawashima & 1 \\
\hline & & DVSVD +EP & 1 \\
\hline & & DVSVI (Cx Fontán) & 1 \\
\hline & & Canal AV Completo & 1 \\
\hline & & CUV (Glenn) & 1 \\
\hline & & Atresia pulmonar (Glenn) & 1 \\
\hline & & SCIH estadio Norwood & 1 \\
\hline & & DVAPT Supracardíaco & 1 \\
\hline & & EP valvular y supravalvular & 1 \\
\hline & & Insuficiencia aortica severa & 1 \\
\hline & & TOF con hemodilución & 1 \\
\hline
\end{tabular}

Referencias: Ao: aorta; CIA: comunicación interauricular; CIV: comunicación interventricular; EP, estenosis pulmonar; AP: arteria pulmonar; DVAPT: drenaje venoso anómalo pulmonar total; AtP: atresia pulmonar; VD: ventrículo derecho; VI: ventrículo izquierdo; DVSVD: doble vía de salida de ventrículo derecho; SCIH: síndrome de corazón izquierdo hipoplásico.

Análisis estadístico: los datos se analizaron mediante el programa estadístico InfoStat ${ }^{\circledR}$.

Diseño estadístico: Las variables categóricas fueron descriptas a partir de sus frecuencias. Los resultados de las variables mensurables se expresaron como media \pm error estándar. Las comparaciones entre grupos se efectuaron a partir de la prueba varianza (ANAVA), un valor de $p<0,05$ fue considerado estadísticamente significativo.

Consideraciones éticas: el plan de trabajo cuenta con la aprobación del Comité de Capacitación y Docencia del Hospital de Niños de la Santísima Trinidad de la Ciudad de Córdoba. (Argentina). Los datos se recolectaron de manera anónima para la inclusión en una base informática, protegida por el secreto estadístico.

\section{Resultados}

Grupo A: 15 p, 6 de sexo femenino y 9 de sexo masculino. Edad media a la intervención: 4,3 años Tiempo de bomba: 78 minutos $(\mathrm{m})$ y tiempo de clampeo: $58 \mathrm{~m}$.
Grupo B. 19 p, 8 de sexo femenino y 11 de sexo masculino. Edad media a la intervención: 1,2 años. Tiempo de bomba: $113,45 \mathrm{~m}$ y tiempo de clampeo: $77,55 \mathrm{~m}$

En este grupo: $11 \mathrm{p}$ tuvieron parada circulatoria, un tiempo promedio de $27 \mathrm{~m}$ (Tabla 2).

\section{Análisis de la Tabla 3}

- Se evidencia una mayor diferencia de $\mathrm{CO}_{2}$ venoarterial durante y al finalizar la CEC.

- En el análisis del pH: se puede apreciar aumento del pH tanto arterial como venoso al inicio de la CEC. A la salida de CEC, se evidencia acidosis venosa.

- El IEO 2 es normal en los valores previos y durante la CEC. Se observa un incremento no significativo post $C E C$, en el grupo $B$.

- Se observa un incremento del ácido láctico intra y sobre todo post bomba en ambos grupos siendo más significativo en el grupo B. (Figura 1).

- Se observa en ambos grupos descenso en los valores de hemoglobina, mayormente en durante el período intraoperatorio. Se evidencia una recupe- 


\begin{tabular}{|c|c|c|c|}
\hline Grupos & $\begin{array}{c}\text { Grupo A } \\
\text { (RASCH } 1 \text { y 2) }\end{array}$ & $\begin{array}{c}\text { Grupo B } \\
\text { (RASCH 3, } 4 \text { y 5) }\end{array}$ & Valor $p$ \\
\hline \multicolumn{4}{|c|}{ Diferencias V-A CO} \\
\hline Basal & 7,43 & 7,48 & 0,2654 \\
\hline Intra & 6,77 & 7,54 & 0,2458 \\
\hline Post & 8,26 & 8,90 & 0,7008 \\
\hline \multicolumn{4}{|l|}{$\mathrm{IEO}_{2}$} \\
\hline Basal & 20,07 & 22,36 & 0,4679 \\
\hline Intra & 21,93 & 22,62 & 0,8424 \\
\hline Post & 22,45 & 27,45 & 0,3750 \\
\hline \multicolumn{4}{|l|}{ pH } \\
\hline Basal & 8,78 & 7,30 & 0,2554 \\
\hline Intra & 7,46 & 7,38 & 0,0380 \\
\hline Post & 7,26 & 7,27 & 0,5315 \\
\hline \multicolumn{4}{|c|}{ Cociente respiratorio: Dif v-a $\mathrm{CO}_{2} / \mathrm{Ca}-\mathrm{vO}_{2}$} \\
\hline Basal & 0,91 & 1,83 & 0,0355 \\
\hline Intra & 1,90 & 1,95 & 0,0102 \\
\hline Post & 1,98 & 3,82 & 0,0385 \\
\hline \multicolumn{4}{|c|}{ Ácido láctico } \\
\hline Basal & 1,03 & 1,18 & 0,2934 \\
\hline Intra & 3,13 & 2,85 & 0,2551 \\
\hline Post & 3,22 & 4,17 & 0,0427 \\
\hline \multicolumn{4}{|c|}{ Hemoglobina } \\
\hline Basal & 10,29 & 12,08 & 0,0122 \\
\hline Intra & 9,49 & 9,35 & 0,8359 \\
\hline Post & 10,64 & 11,49 & 0,1216 \\
\hline
\end{tabular}

Referencia: valor de $\mathrm{p}$ en negrilla $<0,05$.

ración de los valores en el postquirúrgico en ambos grupos.

- En el análisis del cociente respiratorio o gradiente: Difv- $\mathrm{aCO}_{2} / \mathrm{Ca}-\mathrm{vO}_{2}$, se produce un incremento durante y al finalizar la CEC, siendo los mismos significativos en el grupo B.

\section{Discusión}

Adrogué y cols[9] evaluaron el estado ácido base en la falla circulatoria donde la hipoperfusión tisular se manifiesta con hipoxia y acidosis venosa por mayor producción tisular y menor eliminación de $\mathrm{CO}_{2}$ venoso y aumento de valores de lactatos séricos.
En estados de bajo gasto cardíaco, la relación Pv$\mathrm{aCO}_{2}$, es inversamente proporcional al gasto cardíaco, donde el aumento de la misma refleja una reducción progresiva del gasto cardíaco[10],[11].

En nuestra experiencia, se evidencia una mayor diferencia de $\mathrm{CO}_{2}$ veno-arterial durante y al finalizar la CEC, esto se explicaría por disminución de la perfusión tisular con acidosis venosa y bajo flujo para lavar la mayor cantidad de $\mathrm{CO}_{2}$ venoso generado en tejidos, además sus cambios son más precoces que la alteración de lactatos.

En el análisis del pH: se puede apreciar aumento del $\mathrm{pH}$ tanto arterial como venoso al inicio de la CEC, probablemente provocada por la administración de bicarbonato de sodio en la bomba. Diecisiete pacien- 


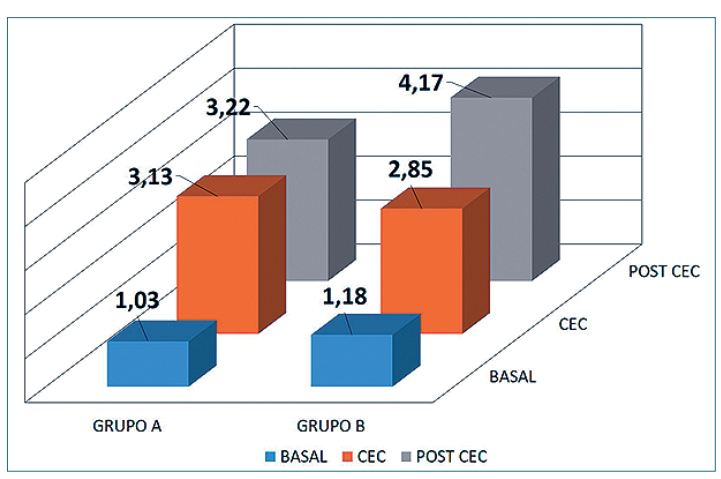

Figura 1. Representación del aumento del ácido láctico en ambos grupos durante la cirugía.

tes de la serie recibieron infusión de bicarbonato en el circuito CEC.

A la salida de CEC, se evidencia acidosis venosa, siendo la misma más notoria en el grupo $B$, la etiología es multifactorial; desde la mayor complejidad quirúrgica, tiempos de bomba y clampeo aórtico más prolongado e influencia de la parada cardiaca circulatoria total.

También puede influir la liberación de $\mathrm{CO}_{2}$ acumulado en los tejidos al iniciarse la recirculación y la administración de bicarbonato en la bomba.

El pH más ácido a nivel venoso central, muestra que el retorno venoso de tejidos mal perfundidos, tienen mayor concentración de $\mathrm{CO}_{2}$, esto genera la salida de protones que al unirse a la hemoglobina desvían la curva de disociación de la hemoglobina a la derecha con mayor liberación de $\mathrm{O}_{2}$ a nivel tisular (Efecto Borh)[6],[10],[11].

El IEO $\mathrm{I}_{2}$ es normal en los valores previos y durante la CEC, probablemente porque el flujo de la bomba es continuo y no pulsátil y por los efectos de la hipotermia y la alcalosis desvían la curva de disociación a la izquierda con mayor afinidad hacia la hemoglobina.

En cambio, una vez finalizada la CEC, aumenta por efectos del recalentamiento y la reperfusión tisular, Además, puede influir la disminución de valores de $\mathrm{SvCO}_{2 v}$ provocadas por síndrome de bajo gasto cardíaco, ventilación inadecuada, fiebre, y dolor[5],[6;[7].

Se puede apreciar una disminución en los valores de la hemoglobina y del contenido arterial y venoso de oxígeno durante la CEC, donde tienen su influencia el sangrado quirúrgico y hemodilución, aumentan las cifras post CEC por corrección del hematocrito con reposición de sangre o glóbulos rojos o por hemoconcentración luego del hemofiltrado/ultrafiltrado.

La saturación arterial de oxígeno $\left(\mathrm{SaO}_{2}\right)$, solo expresa adecuado intercambio de oxígeno a nivel pulmonar, en cambio la saturación venosa $\left(\mathrm{SvCO}_{2}\right)$ es utilizada como un marcador adecuado de oferta/ demanda de oxígeno tisular al igual que las variables derivadas del oxígeno como la extracción de oxígeno tisular $\left(\mathrm{IEO}_{2}\right)[4],[5],[6]$.

Para relacionar la disminución de $\mathrm{SvCO}_{2}$ con bajo gasto cardíaco, se deben descartar la presencia de factores confundidores, como patología respiratoria $\mathrm{o}$ anemia, que disminuyen la $\mathrm{DO}_{2}$ o fiebre y convulsiones que aumentan el $\mathrm{VO}_{2}[4],[5],[6]$.

Los valores de ácido láctico se incrementan durante la CEC por mal perfusión tisular y el aumento postbomba se podría explicar por liberación de lactatos desde la circulación pulmonar durante el recalentamiento y reperfusión. Para ser considerada una acidosis láctica tipo $\mathrm{A}$, se debe asociar a gases con hipoxemia y acidosis, una relación piruvato/lactato elevada y un score inotrópico elevado[12[,[13],[14].

Se debe tener en cuenta la acidosis láctica tipo B provocada por la alteración del aclaramiento de lactatos, la eliminación renal, la función hepática, la infusión de catecolaminas, principalmente adrenali$\mathrm{na}[12],[13],[14],[15]$.

Los valores de lactatos se incrementan durante la CEC por el flujo continuo con menor presión de perfusión tisular y el aumento postbomba podría obedecer a la liberación de lactatos desde la circulación pulmonar en la reperfusión[12],[13].

La ecuación de Fick es útil para comprender al nuevo marcador "cociente respiratorio" o gradiente entre la diferencia veno-arterial de $\mathrm{CO}_{2}$ y el contenido arterio-venoso de oxígeno Cociente respiratorio o gradiente de (Difv-aCO $\left./ \mathrm{Ca}_{2} \mathrm{VO}_{2}\right)[16],[17]$.

Se conoce que el consumo de oxígeno $\left(\mathrm{VO}_{2}\right)$ y la producción tisular de $\mathrm{CO}_{2}\left(\mathrm{VCO}_{2}\right)$, es directamente proporcional al gasto cardíaco y a la entrega de oxígeno $\left(\mathrm{DO}_{2}\right)$ expresada por la diferencia de contenido arterio-venoso de oxígeno (Dif a- $\mathrm{vO}_{2}$ ). O sea durante la homeostasis normal (metabolismo aerobio), la producción de $\mathrm{CO}_{2}\left(\mathrm{VCO}_{2}\right)$, no debe exceder la oferta de oxígeno $\left(\mathrm{DO}_{2}\right)$, la relación $\mathrm{VO}_{2} / \mathrm{DO}_{2}$ no debe ser mayor a 1.

Durante el metabolismo anaerobio se genera un exceso de hidrogeniones $(\mathrm{H}+)$, al no ser utilizados en la fosforilazión oxidativa, el bicarbonato $\left(\mathrm{HCO}_{3}\right)$ amortigua a estos $\mathrm{H}+$ formando ácido carbónico $\left(\mathrm{H}_{2} \mathrm{CO}_{3}\right)$ que se disocia en $\mathrm{CO}_{2}$ y $\mathrm{HO}_{2}$ durante la hidrolisis del ATP, esta mayor producción de $\mathrm{CO}_{2}$ incrementa el numerador del cociente respiratorio[17],[18],[19].

Patel y cols[6], calcula el "cociente respiratorio"en pacientes pediátricos bajo circulación extracorpórea. Ellos demuestran, que el incremento de la relación 
$\mathrm{Pv}-\mathrm{aCO} \mathrm{CO}_{2} / \mathrm{Ca}^{-\mathrm{vO}_{2}}$, refleja metabolismo anaerobio en forma precoz, a diferencia del incremento de lactatos que tiene una cinética más lenta[8].

En nuestra experiencia, el análisis del cociente respiratorio o gradiente de Difv- $\mathrm{aCO}_{2} / \mathrm{Ca}-\mathrm{vO}_{2}$, muestra un incremento al durante y al finalizar la CEC, esto sea explicaría por la acidosis venosa ocasionada por un flujo capilar insuficiente para lavar la mayor cantidad de $\mathrm{CO}_{2}$ venoso generado en tejidos malperfundidos e hipoxémicos.

Si la producción de $\mathrm{CO}_{2}\left(\mathrm{VCO}_{2}\right)$ y el consumo de oxígeno $\left(\mathrm{VO}_{2}\right)$, se mantienen estables, el incremento del índice $\mathrm{v}-\mathrm{CCO}_{2}$ refleja estados de bajo gasto cardía$\mathrm{Co}$, además la disminución del índice $\mathrm{v}-\mathrm{aCO} \mathrm{CO}_{2}$ posterior al tratamiento, refleja una evolución favorable en forma precoz[17],[18].

Si con la fluidoterapia inicial en pacientes en shock, persisten valores de $\mathrm{Pv}^{-a C O} \mathrm{CO}_{2}>6 \mathrm{mmHg}$ a pesar de alcanzar metas de $\mathrm{SvcO}_{2}>70 \%$, refleja alteraciones microcirculatorias que se asocian a una hiperlactacidemia persistente, severa disfunción multiorgánica y mayor mortalidad (valor pronóstico)[18],[19],[20].

\section{Conclusiones}

1- El análisis de las tendencias de las variables derivadas del oxígeno son útiles para evidenciar el equilibrio $\mathrm{DO}_{2} \mathrm{NO}_{2}$, pero se deben interpretar teniendo en cuenta los factores confundidores.

2- La evaluación de los marcadores de perfusión es avalada en numerosas publicaciones en diferentes escenarios de inestabilidad hemodinámica, donde demuestran una gran utilidad para identificar eventos adversos, desenmascarar estados de shock crípticos (shock oculto) y como marcador de evolución.

3- Patel y cols., demuestran la utilidad demuestran la utilidad del nuevo marcador de perfusión "cociente respiratorio" en pacientes pediátricos con cardiopatías congénitas operados bajo circulación extracorpórea, replicando dicha experiencia nosotros encontramos que dicho marcador muestra un valor promisorio en identificar estados de bajo gasto cardíaco con metabolismo anaerobio en forma precoz.

\section{Referencias}

1. Seghaye, MC. The clinical implications of the systemic inflammatory reaction related to cardiac operations in children. Cardiol Young 2003; 13(3):22839. https://doi.org/10.1017/ S1047951103000465

2. Banille, E. Reacción inflamatoria sistémica. Capítulo N¹. Banille E. En: Cardiointensivismo Pediátrico. Fisiología y Decisiones. Páginas: 1-36 Ed RECFOT. 2010

3. Banille, E. Monitoreo Hemodinámico Deductivo. Del Corazón a la Biomonitoreo Cap N 7. En Banille. Monitoreo Cardiopulmonar Pediátrico. De la teoría a la práctica. Páginas: 171-197 Ed REDFOT 2019.

4. Martin J, Shekerdemian LS. The monitoring of venous saturations of oxygen in children with congenitally malformed hearts. Cardiol Young 2009; 19:3439. https://doi.org/10.1017/

\section{S1047951109003539}

5. Banille E, Vittar M, Saenz S, et al. Saturación venosa central de oxígeno. Su valor en el Monitoreo Cardiovascular Pediátrico. Arch Arg Pediatr 2006; 104(5):406411.

6. Patel $\mathrm{R}$, Salonky A, Patel $\mathrm{H}$, et al. Monitoring Microcirculatory Blood Flow during Cardiopulmonary Bypass in Paediatric Cardiac Surgery Patient as Predictor for Anaerobic Metabolism. J Clin Diag Res 2017; 11 :UC22UC25. https://doi.org/10.7860/ JCDR/2017/24360.9721

7. Tibby SM, Murdoch IA. Monitoring cardiac function in intensive care. Arch Dis Child 2003; 88:46-52. https://doi. org/10.1136/adc.88.1.46

8. Bronicki, R. Hemodynamic Monitoring. Pediatric Crit Care Med 2016; 17:S207S214. https://doi.org/10.1097/ PCC.0000000000000779

9. Adrogué HJ, Rashad MN,
Garin AB, et al. Assessing acid-base status in circulatory failure: differences between arterial and central venous blood. NEJM 1989; 320:13121316. https://doi.org/10.1056/ NEJM198905183202004

10. Futier E, Tebould JL, Vallet B. Tissue carbon dioxide measurement as an index of perfusion: Wath have we missed? Trends Anaesth Crit Care 2011; 1:9599. https://doi.org/10.1016/j. tacc.2011.01.012

11. Ospina Tascon GA, Umaña M, Bermudez WF, et al. Can venous to arterial carbon dioxide differences reflect microcirculatory alterations in patients with septic shock? Int Care Med 2016; 42: 211.221. https://doi. org/10.1007/s00134-015-41332

12. Bronicki, RA. Venous oximetry and assessment of oxygen transport balance. Pediatr Crit Care Med 2011; 12:521- 
526. https://doi.org/10.1097/ PCC.0b013e3182211667

13. Joshi $R$, de Witt $B$, Mosler JM. Optimizing oxygen delivery in the critically ill: the utility of lactate and central venous oxygen saturations (SvcO2) as a roadmap of resuscitation in shock. J Emerg Med 2014; 47:493-500. https://doi.org/10.1016/j.jemermed.2014.06.016

14. Bakker, J. Lactate levels and hemodynamics coherence in acute circulatory failure. Best Pract Res Clin Anaesthesiol 2016; 30(4):523-530. https://doi. org/10.1016/j.bpa.2016.11.001

15. Duke, T. Dysoxia and Lactate. Arch Dis Child 1999; 81:343-
350. https://doi.org/10.1136/ adc.81.4.343

16. Banille, E. Intercambio Gaseoso. Cap N¹2. Banille E. En: Cardiointensivismo Pediátrico. Una Mirada Ampliada. Páginas: 295-320. Ed RECFOT 2015

17. Ospina Tascon GA, Bautista Rincon DF, Umañan M, et al. Persistently high venous to arterial carbon dioxide differences during early resuscitation are associated with poor outcomes in septic shock. Crit Care 2013; 17: R294. https://doi.org/10.1186/ cc13160

18. Lamia B, Monett $X$, Tebould $J$. Meaning of arterio-venous $\mathrm{PCO} 2$ difference in circulatory shock. Minerva Anestesiol 2006; 72:597-604

19. Dubin A, Ferrara G, Kannore $\mathrm{E}$, et al. Venoarterial PCO2-toarteriovenous oxygen content difference ratio is a poor surrogate for anaerobic metabolism in hemodilution: an experimental study. Ann Intensive Care 2017; 7:66. https://doi. org/10.1186/s13613-0170288-z

20. Ramesh P, Atul S, Hasmukh P, et al. Monitoring Microcirculatory Blood Flow during Cardiopulmonary Bypass in Paediatrics Cardiac Surgery Patient AS A Predictor for Anaerobic Metabolism.www.jcdr.net 